\title{
Delayed auditory feedback and rhythmic tapping: Evidence for a critical interval shift
}

\author{
STEVEN A. FINNEY \\ Ohio State University, Columbus, Ohio \\ and \\ WILLIAM H. WARREN \\ Brown University, Providence, Rhode Island
}

\begin{abstract}
Delayed auditory feedback (DAF) impairs performance in speech, music, and tapping, with maximal impairment in speech occurring at a delay of about $200 \mathrm{msec}$. This critical interval has played a central role in many explanations of the DAF effect, including both closed-loop feedback explanations and alternative proposals. We investigated the nature of the critical interval in rhythmic tapping-in particular, whether the critical interval has a constant value or is dependent on performance rate. Three experiments in which a synchronization-continuation paradigm was used consistently showed that the critical interval shifted with different tapping rates, with maximal impairment occurring when the delay approximately equaled the tapping rate. We address the implications of these results for theories of DAF.
\end{abstract}

Delayed auditory feedback (DAF), a manipulation in which the sound resulting from a person's actions reaches the ears after a short delay, has been repeatedly shown to disrupt human speech (see Black, 1951, and Lee, 1950, for early reports; see Yates, 1963a, for an early review; see Fabbro \& Daro, 1995, Harrington, 1988, and Zimmerman, Brown, Kelso, Hurtig, \& Forrest, 1988, for more recent work). The impairment demonstrated under DAF includes decreased speech rate, increased loudness, and dysfluencies of various kinds, including syllable repetitions and vowel prolongations. Other tasks are also impaired under DAF, including music performance (Finney, 1997; Gates \& Bradshaw, 1974; Havlicek, 1968), Morse code production (Howell, Powell, \& Khan, 1983; Lee, 1951; Yates, 1965), clapping (Kalmus, Denes, \& Fry, 1955), and rhythmic finger tapping (Chase, Harvey, Standfast, Rapin, \& Sutton, 1961; Finney, 1999; Karlovich \& Graham, 1966; Lee, 1950; Ruhm \& Cooper, 1962). The reported impairment in these other domains is qualitatively similar to that

Portions of the research reported here were contained in the first author's doctoral dissertation in cognitive science at Brown University, supervised by the second author. Experiment 3, as well as additional analyses and manuscript preparation, were completed during S.A.F.'s postdoctoral fellowship at Ohio State University. We thank the thesis readers (Sheila Blumstein, Bruce Kay, and Bruno Repp) for their assistance, support, and advice; we also thank Russ Church, Peter Eimas, Bill Heindel, and Mike Tarr for their assistance, and the attendees of meetings of the New England Sequencing and Timing Association for helpful discussions. Peter Howell, Donald MacKay, Caroline Palmer, and Bruno Repp provided many helpful comments on earlier versions of this paper. Correspondence concerning this article should be sent via email to sf@sfinney.org, or addressed to S. Finney, P. O. box 133, Honolulu, HI 96810. of speech, including a slowing of rate and errors of insertion or repetition; many researchers of DAF (e.g., Chase, Harvey, et al., 1961; Howell et al., 1983; Lee, 1950; MacKay, 1987) have addressed their proposals to both speech and other forms of behavior, such as tapping.

DAF impairment has often been interpreted as evidence for closed-loop feedback control of serial behavior, with auditory feedback being used to control ongoing performance (Chase, 1965; Fairbanks, 1954; Gibson, 1966, p. 36). The impairment then occurs because delayed feedback provides incorrect information about a subject's actions. For example, Lee (1950) proposed that feedback ordinarily provides confirmation that an act has been correctly executed. When there is not timely confirmation of the act (e.g., when the feedback is delayed), the system assumes that an error has occurred and reexecutes the original act, leading to the observed repetition. When the delayed feedback finally arrives, the next item in the sequence can be executed.

One issue with such proposals is that they do not explain a central aspect of the DAF phenomenon: the existence of a delay of maximal impairment, or critical inter$\mathrm{val}$. As has been repeatedly demonstrated, adult speech is maximally disrupted by a delay of about $200 \mathrm{msec}$, with decreasing impairment at either lesser or greater amounts of delay (Black, 1951; Butler \& Galloway, 1957; Fairbanks, 1955; MacKay, 1968). Such a critical interval raises problems for feedback control theories, which would predict that impairment should get worse with increasing delay; for example, according to Lee's (1950) proposal, there should be even more repetitions with longer delays. Such monotonically increasing impairment does not occur as delays are increased. 
One way to explain the decreasing impairment with longer delays within a feedback theory would be to hypothesize that increasing delay tends to cause increased disruption but that feedback must occur fairly close in time to an action to be used as control information. If the feedback information is too distant in time from the action that caused it, the information is not associated with the action and can be ignored. Such a constraint on the binding of perception and action could plausibly be a biological constant that, on the basis of the speech data, would have a value of approximately $200 \mathrm{msec}$ for adults. If the basis of the critical interval is such a fixed value, maximal impairment should occur with a 200-msec delay for any type of behavior-for example, for both speech and tapping. In addition, the critical interval should be constant across different rates of performance.

The effect of performance rate on maximal DAF impairment is relevant to a number of other proposals. As has been noted by Black (1951) and others, the 180-to 200-msec delay interval that causes maximal impairment in speech is approximately the duration of a spoken syllable (reports of mean or median syllable duration in English range from 165 to 220 msec; Black, 1951; Huggins, 1968; Lenneberg, 1967). If there is a direct relationship between syllable duration and DAF impairment, syllable elongation (resulting, e.g., from slowed speech) should lengthen the delay that causes maximal impairment. The effects of rate on the critical interval are also relevant to two recent theories that attempt to explain the impairing effects of DAF. The node structure theory (NST) of MacKay (1987) accounts for the repetitions found under DAF on the basis of a temporal coincidence of the returning feedback and a "hyperexcitable" phase of a mental node involved in the action. This hyperexcitable period occurs at a relatively fixed time after initial node activation (at least for a given type of behavior-e.g., speech), and so the theory predicts that the critical interval should be unaffected by performance rate. On the other hand, the displaced rhythm hypothesis (DRH) of Howell et al. (1983) views DAF disruption as resulting from the temporal coincidence of the onset of the delayed feedback signal and the offset of the undelayed feedback (or the completion of the act itself). Slower performance rates often cause elongation of the units of the performance, which would then require a longer delay for maximal impairment. The DRH thus predicts that critical interval may be influenced by performance rate.

The speech literature contains conflicting reports on the effects of performance rate on DAF impairment. MacKay (1968) tested two different delay values (200 and $263 \mathrm{msec}$ ) with three different rates of speech (maximally fast, slow, and very slow, corresponding to a reported mean syllable duration of 320,500, and 860 msec per syllable when speaking with DAF). Impairment was worse with a 200msec delay than with a $263-\mathrm{msec}$ delay at all rates. This was taken as evidence that the critical interval was unaffected by speech rate. In addition, there were more errors at the faster speech rates. Siegel, Fehst, Garber, and Pick
(1980) had adults perform at normal and rapid speech rates under a range of delays; they reported that a $250-\mathrm{msec}$ delay was maximally disruptive for both speech rates on a duration measure. This result also suggests the absence of a rate effect on critical interval. In contrast, Robinson (1972) used a task in which subjects counted aloud to a pacing signal that varied in rate across trials. There was an effect of performance rate on the delay causing maximal impairment, with longer delays being required to cause maximal impairment at slower rates. Finally, Zanini, Clarici, Fabbro, and Bava (1999) tested a single delay of $200 \mathrm{msec}$ with Italian speakers speaking at two different rates; overall impairment was significantly less at the faster rate, contrasting with MacKay (1968). The conflicts among these four studies suggest that rate effects on critical interval in speech are an unresolved empirical question.

Research on the critical interval in tapping and music performance has not addressed performance rate. Ruhm and Cooper (1962) reported that a 200-msec delay gave more impairment in a tapping task than did a 100-, 300-, or 400-msec delay, but tapping rate was not controlled. Howell et al. (1983) found maximal impairment (using a timing measure) for error-free Morse code transmission at a delay near $200 \mathrm{msec}$. The $200-\mathrm{msec}$ critical interval reported in these studies matches the value for speech, supporting the view that this might be a universal DAF constant. However, Gates, Bradshaw, and Nettleton (1974) reported that maximal impairment in musical keyboard performance occurred with a delay of $270 \mathrm{msec}$, but in neither this study nor other DAF studies on music (e.g., Finney, 1997) was performance rate controlled as a factor.

The present research focuses on the question of whether the DAF critical interval depends on production rate, using a rhythmic tapping task. Tapping allows for precise control over rate and may, therefore, be a better domain for the investigation of production rate effects than is speech. In speech, the signal changes dramatically from moment to moment, and rate changes in speech necessarily affect some aspects of the speech signal (e.g., vowels) more than others. In addition, syllables are not of equal length, so the relation between the arrival of the delayed feedback and a given syllable cannot be precisely controlled. With tapping, however, each executed finger movement is approximately identical, and initial tapping rate can be easily controlled by using a synchronizationcontinuation paradigm (Wing \& Kristofferson, 1973).

We report three experiments in which the following two questions were addressed. (1) Is there a DAF critical interval in tapping, and if so, what is its value? A critical interval of $200 \mathrm{msec}$ in tapping would provide an important replication of the results for speech; the absence of a critical interval might indicate important differences between the two domains. (2) Does the critical interval for tapping have a constant value, or is it dependent on rate? A fixed critical interval would be consistent with one form of feedback-based explanation for the DAF effect, as well as with MacKay's (1987) NST; a rate-dependent critical interval would require an alternative explanation. 


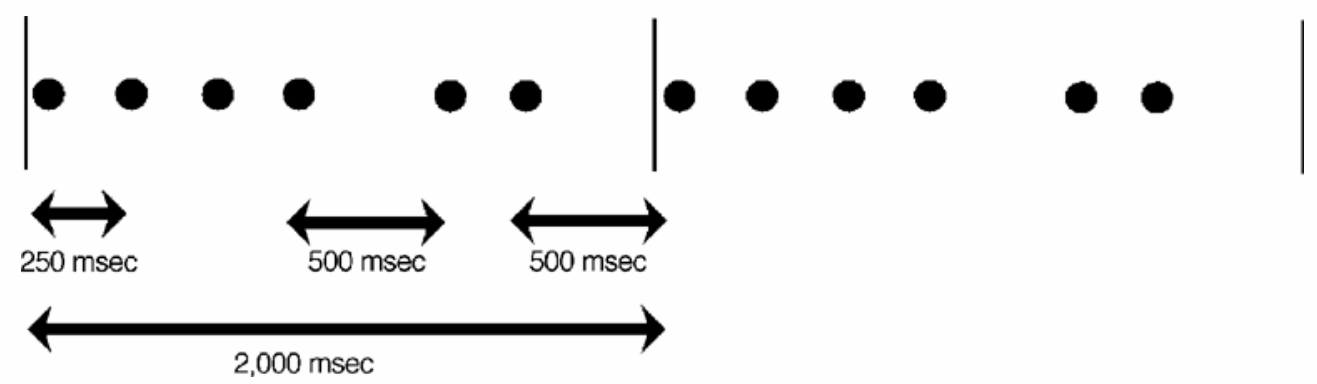

Figure 1. Tapping pattern for Experiments 1 and 3, 250-msec rate. Filled circles indicate a tap; vertical bars indicate the beginning of the pattern.

\section{EXPERIMENT 1}

In Experiment 1, the effect of various delays on the tapping of a simple rhythmic pattern was investigated. Two different production rates were used, and rate was controlled with a synchronization-continuation paradigm.

\section{Method}

\section{Subjects}

Eighteen Brown University undergraduates participated in Experiment 1 ; they received either payment or course credit for their participation.

\section{Equipment}

The subjects tapped on a single white key of a Fatar Studio 49 electronic MIDI (Music Instrument Digital Interface) keyboard. The MIDI output of the keyboard was connected to a Silicon Graphics Indigo computer, and a custom program (see Finney, 2001) recorded all incoming data (keypress time, key release time, note value, and keypress velocity). The program also generated auditory output by sending MIDI data to a Yamaha TX81Z digital tone generator; the MIDI output could be delayed relative to the input keystroke. The TX81Z produced pure tones, which the subjects listened to through Koss PRO/4XTC headphones.

The subject's forearm was supported by a padded wrist rest that was adjusted for his or her comfort. View of the tapping hand was blocked.

\section{Stimuli and Delay Conditions}

The pattern used for tapping in Experiment 1 was a group of 4 taps followed by a group of 2 taps; see Figure 1 for a diagram of this pattern at the 250-msec rate. This will be referred to as the 4-2 pattern; it has been used in previous DAF tapping studies (e.g., Karlovich \& Graham 1966, 1967; Ruhm \& Cooper, 1962), in which it showed more marked effects than did simpler patterns, such as groups of 3 (see Experiment 2 in the present paper for confirmation) or a 3-1 pattern. ${ }^{1}$ The pattern was tapped at two rates; the $250-$ msec rate had intertap onset intervals of $250 \mathrm{msec}$ within a group (within-group interval) and $500 \mathrm{msec}$ between groups (betweengroup interval), whereas the 400 -msec rate had a within-group interval of $400 \mathrm{msec}$ and a between-group interval of $800 \mathrm{msec}$.

A pilot study reported in Finney (1999), in which tapping rates of 250,350 , and $450 \mathrm{msec}$ were used, provided preliminary evidence that the delay causing maximal impairment occurred in the neighborhood of the tapping rate. For Experiment 1, a range of delay values near each tapping rate was used to explore the critical region more precisely. At the 250-msec tapping rate, the delays used were $100,150,200,225,250,275,300,350,400$, and $450 \mathrm{msec}$, as well as synchronous feedback (which will also be referred to as a
0 -msec delay) — a total of 11 conditions. At the 400-msec rate, the delays chosen were $0,200,250,300,350,375,400,425,450,475$, 500 , and $550 \mathrm{msec}$, as well as synchronous feedback-a total of 12 conditions.

\section{Procedure}

In the synchronization- continuation paradigm, subjects initially tap along with a pacing signal (the synchronization phase); the pacing tones then stop, and the subjects continue tapping at the same pattern and rate (the continuation phase). In Experiment 1, the synchronization signal was the 4-2 pattern presented with a digitized pure tone of $1175 \mathrm{~Hz}$ (the note $\mathrm{D}^{6}$ ) of $30-\mathrm{msec}$ duration. Four full patterns were presented for synchronization in the 250 -msec rate condition; at the slower 400-msec rate, only three synchronization patterns were presented (this kept the slower trials to a reasonable length). There was no auditory feedback to the subject's taps during the synchronization phase, but feedback (synchronous or delayed) started immediately after the synchronization patterns. The subjects continued tapping without a pacing signal for a time sufficient for 7.5 patterns (equated for number of taps in the two rate conditions). Trials were $23 \mathrm{sec}$ long in the $250-\mathrm{msec}$ rate condition and $34 \mathrm{sec}$ long in the 400-msec rate condition.

The subjects were asked to tap with the index finger of their (selfreported) dominant hand on a particular white key of the keyboard, to tap from the wrist, and not to lift their finger from the key. In order to avoid biasing the subjects toward a particular method of generating the pattern, nothing about the pattern structure or the number 4 or 2 was mentioned in the instructions. The written instructions also explicitly suggested that the subjects should try to ignore the sounds if they found them disruptive.

Tones of different pitch and duration were used to clearly differentiate the pacing and feedback signals. The feedback tones were 100 -msec duration tones of $660 \mathrm{~Hz}$ (the note $\mathrm{E}_{5}$ ); they were quite distinct from the higher pitched pacing signals. The pitch, loudness, and duration of feedback tones were not affected by the subject's keystrokes. Sound levels were set to allow feedback and pacing tones to be heard simultaneously (as occurred during practice sequences); pacing tones were $79 \mathrm{~dB}$, and feedback tones (lower pitched, but longer) were $78 \mathrm{~dB}$, based on measurements taken with a Radio Shack sound-level meter held 1/4 in. away from the headphone speaker. Masking white noise was also presented over the headphones to block any synchronous auditory feedback resulting from physical key noise. The white noise was on from the very start of the trial and stopped after the designated trial duration; this was the subject's cue that the trial had ended. ${ }^{2}$

The experimental trials were preceded by three practice sequences. First, the subjects tapped along with the pacing pattern, hearing synchronous feedback, until the experimenter felt they were comfortable with the task (typically, 25-50 sec). Next, the subjects practiced tapping along with the pacing pattern without auditory 

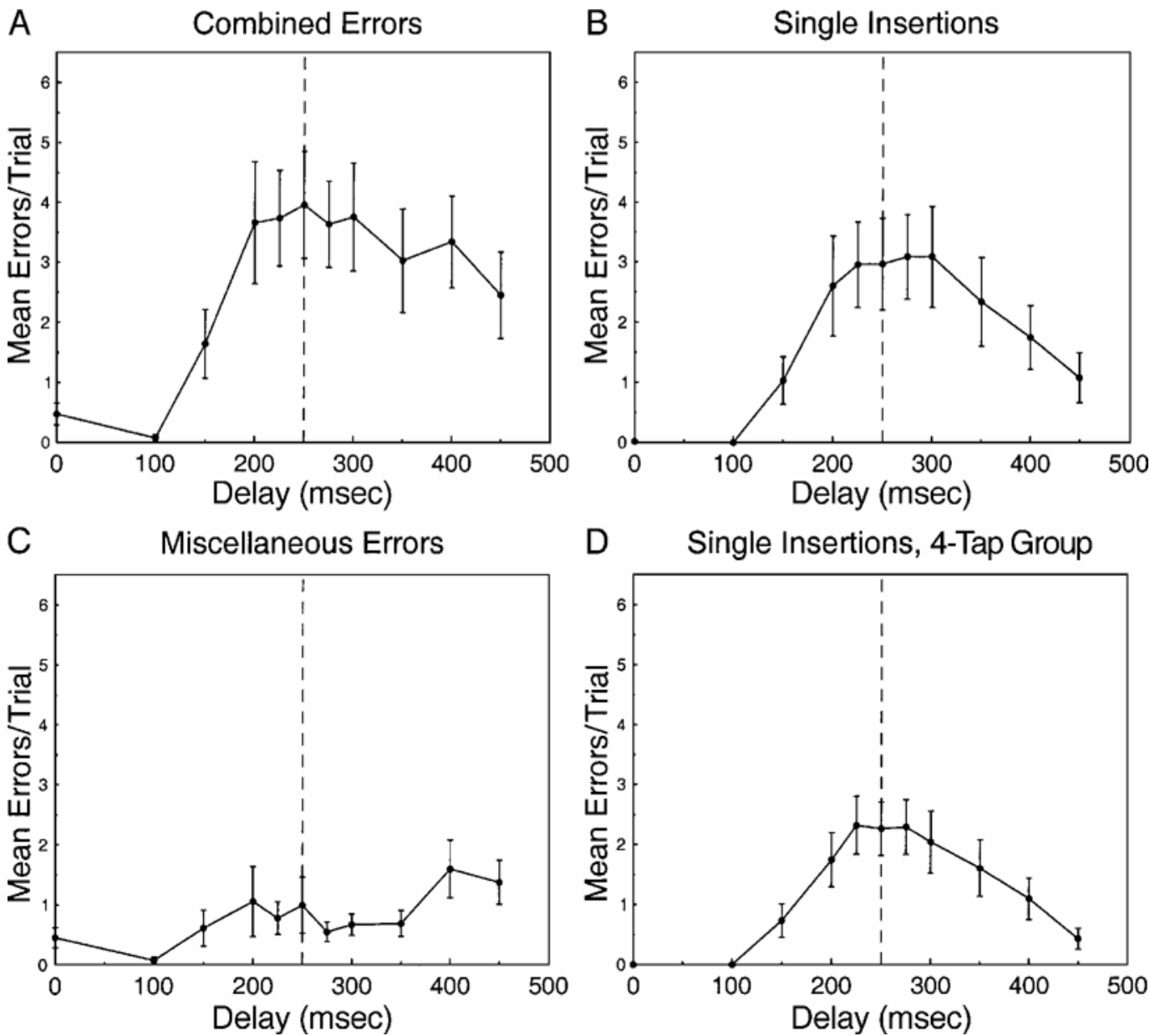

Figure 2. Experiment 1, 250-msec tapping rate (indicated by the vertical dashed line): mean errors per trial (with standard error bars) for different categories of errors.

feedback. Finally, the subjects performed one practice continuation trial, which was identical to a test trial in the 0-msec delay (synchronous feedback) condition.

Trials were blocked by tapping rate; half the subjects received the 250-msec block first, and half received the 400-msec block. Within a block, the subjects performed three trials at each delay; the trials in each block were independently randomized for each subject. A short break was given in the middle of each block, and a longer break was given between blocks; in addition, the subjects were permitted to rest at any point.

\section{Results}

Filtering of the data was performed as follows. Trials in which a subject was not synchronized on all 6 taps of the final synchronization pattern (based on visual inspection of a graphical representation of each trial) were removed from further analysis; this comprised $2 \%$ of all the trials. In the remaining trials, taps that were brushes of an adjacent key (i.e., when one keystroke followed another by less than $20 \mathrm{msec}$ ) were removed from the data. Finally, because the trials ended at a fixed time, a group of taps could be interrupted before it was complete; since this was not a subject error, the final group of taps in each trial was removed from further analysis.

\section{Error Measures and Critical Interval}

One measurement of impairment used in DAF tapping studies (e.g., Chase, Harvey, et al., 1961; Ruhm \& Cooper, 1962) is counting or number errors: how frequently groups in a pattern are performed with an incorrect number of taps. To calculate such measures, it is necessary to have a criterion for which taps should be considered part of a single group. In the patterns used here, the time interval that separated the groups was always twice the time interval separating taps within a group; the mean of these two values was used as the cutoff criterion. For instance, at the 250-msec tapping rate, taps separated by interonset times of less than $375 \mathrm{msec}$ were classified as belonging to the same group of taps, whereas an interonset time greater than or equal to $375 \mathrm{msec}$ was taken as a separator between groups.

Correct execution of the sequence would consist of alternating groups of 4 and 2 taps. A given group may be in 
A

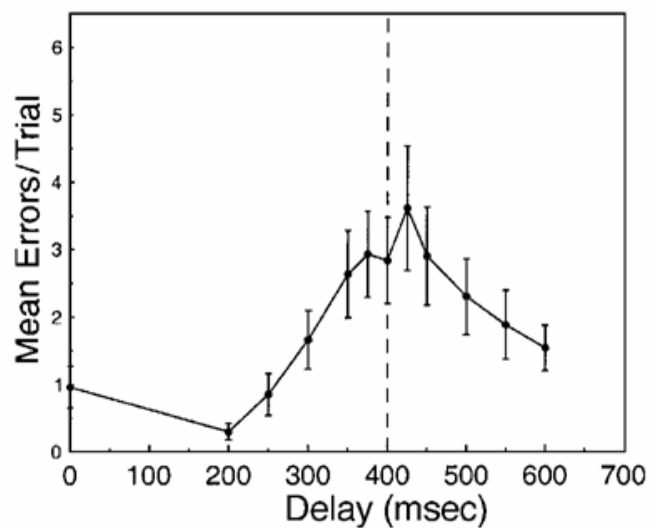

C Miscellaneous Errors

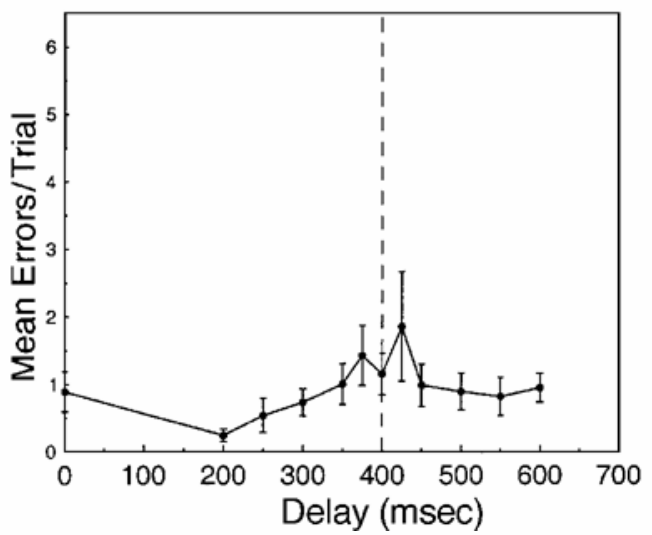

B

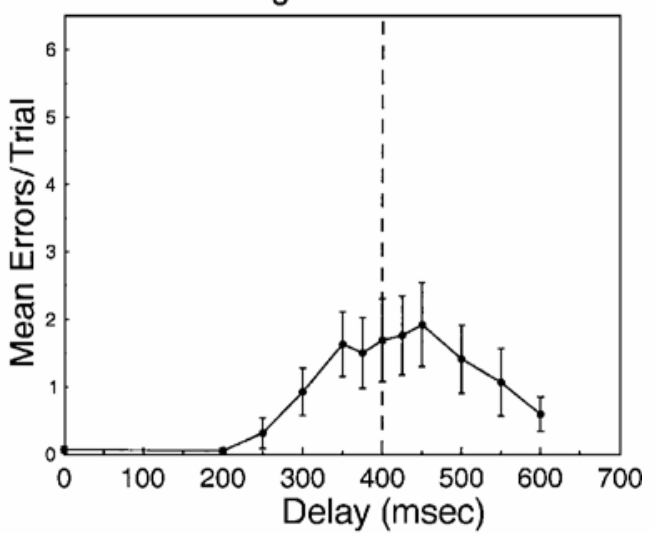

D Single Insertions, 4-Tap Group

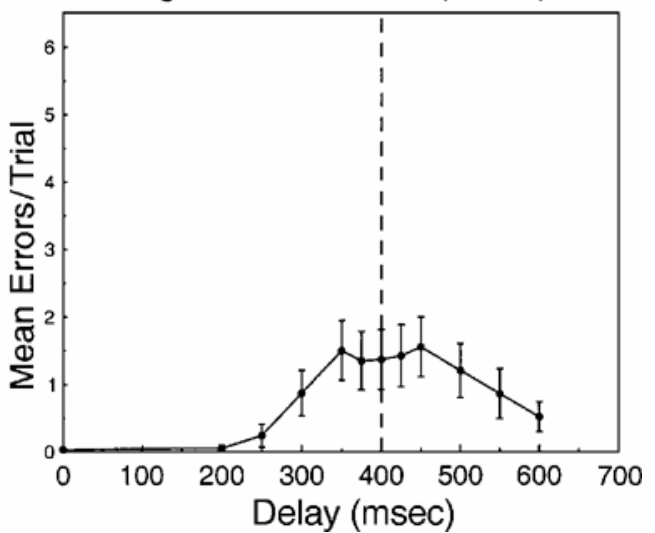

Figure 3. Experiment 1, 400-msec tapping rate (indicated by the vertical dashed line): mean errors per trial (with standard error bars) for different categories of errors.

error because one or more taps were omitted (deleted) or because one or more taps were inserted (added); such inserted taps are a form of repetition, analogous to the repetitions found in speech. Data for the following error measures are presented in Figure 2 for the 250-msec tapping rate and in Figure 3 for the 400-msec rate; these data are the mean number of groups in error per trial across subjects.

1. Combined errors (Figures 2A and 3A): any group that did not include either 4 or 2 taps was counted as an error (i.e., all groups with either deleted or inserted taps). ${ }^{3}$

2. Single insertions (Figures $2 \mathrm{~B}$ and $3 \mathrm{~B}$ ): groups containing a single extra tap. ${ }^{4}$ As can be seen by comparing panels $A$ and $B$ in the figures, such single insertions are the predominant error type in Experiment 1.

3. Miscellaneous errors (Figures 2C and 3C): all counting errors except for single insertions. This includes all deletions (including isolated single taps), as well as insertions of more than one tap. By definition, the combinederrors measure is the sum of single insertions and miscellaneous errors.

4. 4-tap group insertions (Figures 2D and 3D): groups of 5 taps (i.e., insertion errors on the 4-tap group of the pattern). Comparison of these to the corresponding panel B in each of the figures shows that most insertion errors occurred on the first (and longer) group.

Analysis of the 250-msec rate. At the 250-msec tapping rate, all the measures except for miscellaneous errors showed a peak of impairment (i.e., a critical interval) near $250 \mathrm{msec}$, with the insertion measures showing a broad peak ranging from about 225 to $300 \mathrm{msec}$. The differentially impairing effect of the delays was confirmed with one-way within-subjects analyses of variance (ANOVAs) performed on each of the four error measures, with delay time as the single factor. The effect of delay time was significant for the combined-errors measure $[F(10,170)=$ $9.18, p<.001]$, the overall single-insertions measure $[F(10,170)=9.11, p<.001]$, the single-insertion 4-tap group measure $[F(10,170)=11.46, p<.001]$, and even the miscellaneous errors measure $[F(10,170)=1.94, p<$ $.05]$. The predominant error type was single insertions, with $70 \%$ of the total errors being such single insertions. Of these single insertions, $69 \%$ occurred on the 4-tap group (that is, tapping a group of 5). Further details on error subtypes are provided in Table 1.

Analysis of the 400-msec rate. At the 400-msec tapping rate, all the measures showed a peak of impairment (i.e., a critical interval) near $400 \mathrm{msec}$, with the insertion 
Table 1

Percentages of Error Subtypes for Each Tapping Rate (Collapsed Across Delay Values)

\begin{tabular}{|c|c|c|c|c|c|c|}
\hline & \multicolumn{2}{|c|}{$\begin{array}{l}\text { Experiment } 1 \\
(4-2 \text { Pattern })\end{array}$} & \multicolumn{2}{|c|}{$\begin{array}{c}\text { Experiment } 2 \\
\text { (3 Pattern) }\end{array}$} & \multicolumn{2}{|c|}{$\begin{array}{l}\text { Experiment } 3 \\
(4-2 \text { Pattern }) \\
\end{array}$} \\
\hline & $250 \mathrm{msec}$ & $400 \mathrm{msec}$ & $250 \mathrm{msec}$ & $400 \mathrm{msec}$ & $250 \mathrm{msec}$ & $400 \mathrm{msec}$ \\
\hline Mean Total Errors (Count) & 2.70 & 2.03 & 1.31 & 0.92 & 2.70 & 1.34 \\
\hline \multicolumn{7}{|l|}{ Error subtypes (\%) } \\
\hline Single insertions (long group) & 49 & 45 & - & - & 51 & 36 \\
\hline Single insertions (short group) & 21 & 8 & - & - & 19 & 7 \\
\hline Single insertions (combined) & 70 & 53 & 49 & 22 & 70 & 43 \\
\hline Double insertions (long group) & 6 & 15 & 0 & 1 & 9 & 22 \\
\hline Single deletions (long group) & 5 & 6 & 19 & 25 & 6 & 7 \\
\hline Isolated taps & 15 & 15 & 30 & 41 & 13 & 11 \\
\hline Other & 4 & 11 & 2 & 11 & 2 & 17 \\
\hline
\end{tabular}

measures showing a broad peak ranging from about 350 to $450 \mathrm{msec}$. One-way within-subjects ANOVAs were performed on each of the four error measures for the 400msec tapping rate, with delay time as the single factor. The effect of delay time was significant for the combinederrors measure $[F(11,187)=7.19, p<.001]$, the overall single-insertions measure $[F(11,187)=5.79, p<.001]$, the single-insertion 4-tap group measure $[F(11,187)=$ $6.12, p<.001]$, and even the miscellaneous errors measure $[F(11,187)=1.94, p<.05]$. A further breakdown of error types is shown in Table 1.

Rate effects. An inspection of the graphs indicates that the critical interval is different at the two rates, but this important result must be more rigorously established. Two statistical approaches were taken to assess the apparent difference in critical interval at the two rates. First, a two-way ANOVA on the combined-errors measures, with delay and tapping rate as factors, was performed to determine whether the effect of the different delays varied with rate; only the delay values shared across both rates $(0$, $200,250,300,350,400$, and $450 \mathrm{msec}$ ) were used in the analysis. The main effect of tapping rate was significant $[F(1,17)=8.06, p<.05]$, showing that there were more errors at the faster tapping rate. The main effect of delay was also significant $[F(6,102)=5.76, p<.001]$, showing that the different delays caused different amounts of impairment. Most important, the interaction of rate and delay was significant $[F(6,102)=9.335, p<.001]$, confirming that the effect of the different delays depended on tapping rate.

The presence of an interaction, however, does not by itself say anything about the maxima of the impairment curves (i.e., the critical interval). Owing to the obvious skew of the curves in Figures 2 and 3 (particularly the 0msec value), a simple quadratic curve fit was not appropriate. Therefore, to assess whether the maxima at the two rates were statistically different, we determined the delay value that caused maximal impairment for each subject at each of the two rates (if there was more than one delay causing the same maximum error for a subject, the mean of these delays was used). These per subject maxima were then entered into a one-way ANOVA, with tapping rate as the single factor. The effect was significant $[F(1,17)=$
$11.50, p<.005$ ], with the mean maximally impairing delay being $274 \mathrm{msec}$ at the 250 -msec tapping rate and $377 \mathrm{msec}$ at the $400-\mathrm{msec}$ tapping rate. These results demonstrate that the critical interval is dependent on tapping rate; it is of further interest to note that the critical interval value is close to the tapping rate.

\section{Timing of Insertions}

One additional observation is that the timing of taps in groups that contained an extra tap maintained the underlying beat fairly closely, despite the fact that an error had been made. That is, insertion errors were in time; they were not simply inserted at random intervals. For correctly tapped 4-tap groups in the 250-msec condition (collapsed across subjects and delay values), the overall mean time between tap onsets (intertap interval, or ITI) was $253 \mathrm{msec}$, whereas the mean time preceding the final tap (i.e., between the onsets of the 3rd and 4th taps) was $252 \mathrm{msec}$. In 5-tap groups with an insertion error, the overall mean ITI was $249 \mathrm{msec}$, whereas the mean time preceding the final (inserted) tap (i.e., between the onsets of the 4th and 5th taps) was 247 msec. In the 400 -msec condition, correctly tapped 4-tap groups had an overall mean ITI of $387 \mathrm{msec}$, and the mean time preceding the final tap was also $387 \mathrm{msec}$. The 5 -tap groups had an overall mean ITI of $377 \mathrm{msec}$, and the mean time preceding the final (inserted) tap was $376 \mathrm{msec}$. Although there were small differences, the underlying tapping rate was well maintained even in the case of an insertion error.

\section{Discussion}

The data provide clear evidence of a shift in the critical interval that depends on tapping rate. At the 250msec rate, maximal impairment occurred at a delay near $250 \mathrm{msec}$ (with a range of about $\pm 50 \mathrm{msec}$ ), whereas at the 400-msec rate, maximal impairment occurred with a delay near $400 \mathrm{msec}$. At both rates, there was clear evidence for a critical interval, albeit one with a value different from the 200-msec critical interval usually cited for speech, and also different from the earlier report (Ruhm $\&$ Cooper, 1962) of a 200-msec critical interval in tapping. It is of particular interest that the critical interval was approximately equal to the tapping rate. 


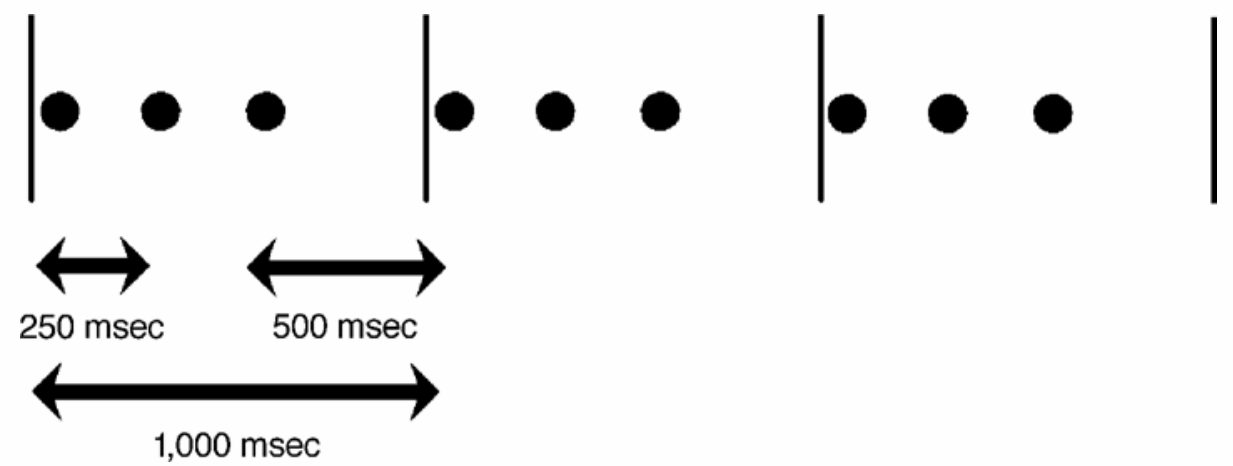

Figure 4. Tapping pattern for Experiment 2, 250-msec rate. Filled circles indicate a tap; vertical bars indicate the beginning of the pattern.

\section{EXPERIMENT 2}

One concern with Experiment 1 is that the results might be specific to the particular 4-2 pattern used. In addition, quantitative analysis on such a two-part pattern involves a number of complexities and ambiguities. Experiment 2 was designed to replicate the critical interval shift results of Experiment 1 with a simpler pattern employing groups of 3 taps; such a 3-tap pattern has been used in previous DAF work (e.g., Chase, Harvey, Standfast, Rapin, \& Sutton, 1959, 1961).

\section{Method}

\section{Subjects}

Eighteen Brown University undergraduates participated in this experiment; they received either payment or course credit for their participation.

\section{Procedure}

Experiment 2 used the same equipment and procedure as Experiment 1 , with the following exceptions. First, a pattern of 3 taps was used in place of the 4-2 pattern; see Figure 4 for a diagram of this pattern at the 250 -msec rate. Second, because of the shorter pattern, more pacing patterns were provided during the synchronization phase. At the $250-\mathrm{msec}$ rate, there were six synchronization patterns, and continuation time was allowed for 15 repetitions of the pattern, resulting in a total trial duration of $21 \mathrm{sec}$. At the 400 msec rate, there were four synchronization patterns, and time was again allowed for 15 repetitions, giving a total trial duration of $30 \mathrm{sec}$. The same delays as those in Experiment 1 were used for each tapping rate, ranging from 100 to $450 \mathrm{msec}$ for the 250-msec tapping rate, and from 200 to $550 \mathrm{msec}$ for the $400-\mathrm{msec}$ tapping rate, with an additional synchronous feedback condition for both rates.

The continuation phase of Experiment 2 contained the same number of total groups as Experiment 1, although Experiment 1 used a two-part pattern. Since the results are presented as the mean number of groups in error per trial, direct comparisons with the data of Experiment 1 can be made.

\section{Results}

For brevity, only the combined-errors measure will be presented and analyzed in detail, although additional information on error subtypes is shown in Table 1. The combined-errors data are shown in Figure 5.
As can be seen by an inspection of Figure $5 \mathrm{~A}$, at the 250msec rate, a maximum occurred for combined errors at the 275-msec delay. A one-way within-subjects ANOVA was performed on the combined error data, with delay time as the single factor; the effect of delay time was significant $[F(10,170)=4.89, p<.001]$.

At the 400-msec rate, an inspection of Figure 5B shows that maximal impairment occurred at the 400-msec delay. A one-way within-subjects ANOVA was performed on the combined-errors data, with delay time as the single factor. The effect of delay time was only marginally significant $[F(11,187)=1.77, p=.063]$; the variance in the data was very high, although a peak is clear in the impairment curve.

\section{Rate Effects}

The same two statistical approaches as those in Experiment 1 were taken to assess the apparent difference in critical interval at the two tapping rates. First, a two-way ANOVA on the combined-errors measures, with delay and tapping rate as factors, was performed (with the analysis restricted to the delay values shared across tapping rates). The main effect of tapping rate was significant $[F(1,17)=5.54, p<.01]$, with a higher incidence of errors at the faster tapping rate. The main effect of delay was also significant $[F(6,102)=3.01, p<.001]$. Most important, the interaction of rate and delay was significant $[F(6,102)=2.55, p<.05]$, confirming that the effect of the different delays depended on tapping rate.

An ANOVA using the individual subject maxima as the dependent variable and tapping rate as the single factor confirmed that the maximum shifted with tapping rate $[F(1,17)=15.62, p<.01]$. The mean maximally impairing delay was $275 \mathrm{msec}$ at the 250 -msec tapping rate and $360 \mathrm{msec}$ at the $400-\mathrm{msec}$ tapping rate. These values are close to the tapping rate, as they were in Experiment 1.

\section{Pattern Effects}

A comparison of Figures $5 \mathrm{~A}$ and $5 \mathrm{~B}$ with the corresponding data from Figures $2 \mathrm{~A}$ and $3 \mathrm{~A}$ suggests that the overall error rate is lower for the 3-tap pattern than for the 4-2 pattern of Experiment 1 (recall that errors were 
A

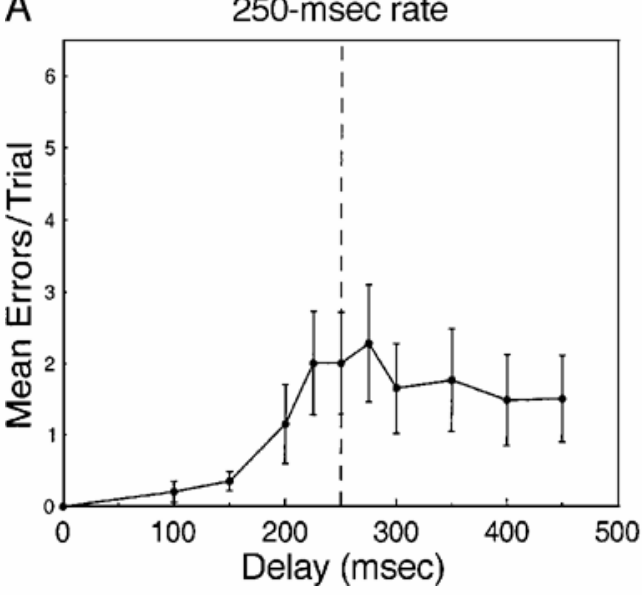

B

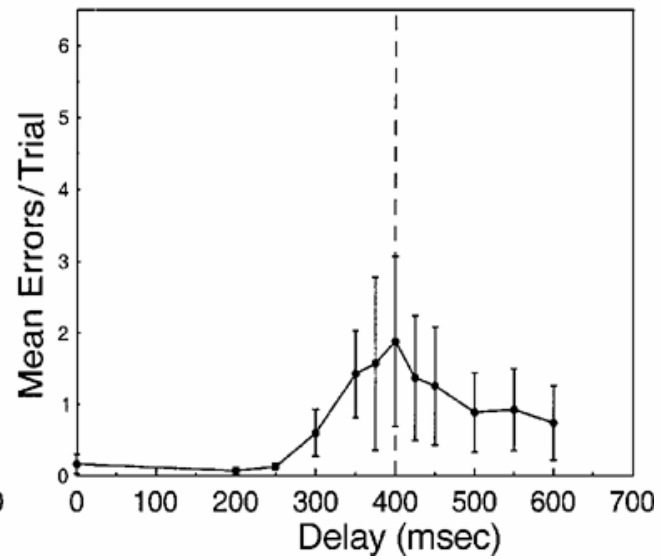

Figure 5. Experiment 2: mean combined errors per trial (with standard error bars).

calculated as the mean number of groups in error per trial, and that there were an equal number of groups per trial in both experiments). An ANOVA was performed for each rate, using combined errors as a dependent variable and experiment as the single factor. At the 250-msec rate, the mean combined-errors count was 2.70 for Experiment 1 and 1.33 for Experiment $2[F(1,34)=3.21, p=$ $.08]$. At the 400-msec rate, the mean combined-errors count was 2.04 for Experiment 1 and 0.91 for Experiment $2[F(1,34)=2.74, p=.11]$. Although there was a clear trend toward greater impairment with the longer 2tap group pattern, it was only marginally significant.

\section{Timing of Insertions}

Analysis of the timing of correct groups and insertion groups was performed as in Experiment 1. For correctly tapped 3-tap groups in the 250-msec condition (collapsed across subjects and delay values), the overall mean time between tap onsets (ITI) was $261 \mathrm{msec}$, whereas the mean time preceding the final tap (i.e., between the onsets of the 2 nd and 3rd taps) was 259 msec. In 4-tap groups (an insertion error), the overall mean ITI was $258 \mathrm{msec}$, whereas the mean time preceding the final (inserted) tap was $252 \mathrm{msec}$. In the $400-\mathrm{msec}$ condition, correctly tapped 3-tap groups had an overall mean ITI of $403 \mathrm{msec}$, and the mean time preceding the final tap was $400 \mathrm{msec}$. The 4tap groups had an overall mean ITI of $398 \mathrm{msec}$, and the mean time preceding the final (inserted) tap was $386 \mathrm{msec}$. Although there were small differences, the underlying tapping rate was again well maintained even in the case of error.

\section{Discussion}

The results of Experiment 2 confirm the shift in the critical interval with tapping rate that was found in Experiment 1 , using a different tapping pattern. The overall error rates were lower than those in Experiment 1, although the differences were not statistically significant.
Of primary interest is that even with the different pattern and the lower error rate, there was still evidence for a critical interval that matched the tapping rate.

\section{EXPERIMENT 3}

In Experiments 1 and 2, the range of delays used was different for the two tapping rates, and the mean of the delays at each rate was, in fact, close to the tapping rate itself. A possible concern is adaptation-level effects. In perceptual experiments, the response of a subject to a given set of stimuli has been shown to be affected by the central tendency of that set of stimuli (Helson, 1964). It is possible that similar adaptation effects could occur in motor tasks and that the apparent equivalence of critical interval to the tapping rate is an artifact of using a different range of delay intervals at the two rates. To control for this possibility, Experiment 3 replicated Experiment 1, but with the same set of delays (ranging from 0 to $550 \mathrm{msec}$ ) at both tapping rates.

\section{Method}

\section{Subjects}

Eighteen Ohio State University undergraduates, enrolled in an introductory psychology course, participated in this experiment. They received course credit for their participation.

\section{Procedure}

Because the 4-2 pattern used in Experiment 1 provided higher error rates than the 3-tap pattern of Experiment 2, the 4-2 pattern was used for Experiment 3. The tapping rates were again 250 and $400 \mathrm{msec}$, but delay values of $0,100,150,200,250,300,350,400$, 450,500 , and $550 \mathrm{msec}$ were used at both tapping rates. The procedure was identical to that of Experiment 1, except that a fourth practice trial with a $300-\mathrm{msec}$ delay was added to the initial practice sequence; this gave the subjects one experience with DAF before the actual experimental trials.

Experiment 3 used the same keyboard and tone generator as Experiments 1 and 2. Keystroke data was collected and manipulated with the FTAP program (Finney, 2001) on a Pentium-based PC. The subjects listened to the stimuli with AKG K270 headphones. 

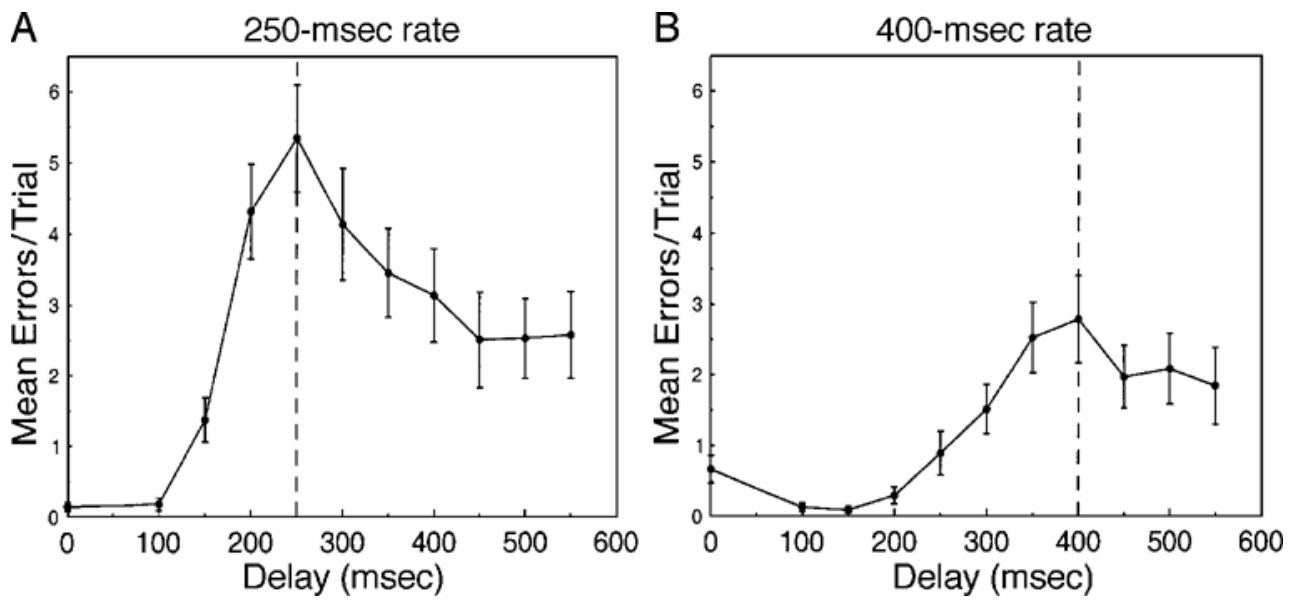

Figure 6. Experiment 3: mean combined errors per trial (with standard error bars).

\section{Results}

For brevity, only the combined-errors measure will be analyzed; these data are presented in Figure 6. Additional information on error subtypes is provided in Table 1 .

As can be seen by inspection of Figure 6A, at the 250msec rate, maximal impairment occurred with a 250 msec delay. A one-way within-subjects ANOVA was performed on the combined-errors data, with delay time as the single factor. The effect of delay time was significant $[F(10,170)=18.16, p<.001]$.

At the 400-msec rate, an inspection of Figure 6B shows that maximal impairment occurred at a 400-msec delay. A one-way within-subjects ANOVA was performed on the combined-errors data, with delay time as the single factor. The effect of delay time was significant $[F(10,170)=10.21, p<.001]$.

One additional aspect of the data in Figure 6A is worth noting. At the 250-msec rate, the delays which are approximately twice the $250-\mathrm{msec}$ tapping rate (i.e., $450-$, 500-, and 550-msec delays) do not show any signs of increased impairment. That is, there is not a second peak of impairment at an integer multiple of the tapping rate.

\section{Rate Effects}

To assess the apparent difference in critical interval at the two rates, a two-way ANOVA on the combinederrors measures, with delay and rate as factors, was performed. The main effect of rate was significant $[F(1,17)=$ $15.96, p=.001]$, indicating a higher incidence of errors at the faster tapping rate. The main effect of delay was also significant $[F(10,170)=14.86, p<.001]$. Most important, the interaction of rate and delay was significant $[F(10,170)=15.61, p<.001]$, confirming that the effect of the different delays depended on tapping rate.

An ANOVA using the individual subject maxima as the dependent variable and tapping rate as the single factor confirmed that the maximum shifted with tapping rate $[F(1,17)=16.89, p=.001]$. The mean maximally im- pairing delay was $253 \mathrm{msec}$ at the 250 -msec tapping rate and $376 \mathrm{msec}$ at the $400-\mathrm{msec}$ tapping rate. These values are once again close to the tapping rate, as they were in Experiments 1 and 2.

\section{Timing of Insertions}

Analysis of the timing of correct groups and insertion groups was performed as in Experiment 1. For correctly tapped 4-tap groups in the 250-msec condition (collapsed across subjects and delay values), the overall mean time between tap onsets (ITI) was $253 \mathrm{msec}$, whereas the mean time preceding the final tap (i.e., between the onsets of the 3rd and 4th taps) was $256 \mathrm{msec}$. In 5-tap groups with an insertion error, the overall mean ITI was $251 \mathrm{msec}$, whereas the mean time preceding the final (inserted) tap was $252 \mathrm{msec}$. In the 400-msec condition, correctly tapped 4-tap groups had an overall mean ITI of $386 \mathrm{msec}$, and the mean time preceding the final tap was $392 \mathrm{msec}$. The 5-tap groups had an overall mean ITI of $378 \mathrm{msec}$, and the mean time preceding the final (inserted) tap was $366 \mathrm{msec}$. Although there were small differences, the underlying tapping rate was well maintained even in the case of error.

\section{Discussion}

The results demonstrate that the critical interval shift observed in Experiments 1 and 2 was not due to the fact that the delay values in those experiments had a mean value close to the tapping rate. The result was replicated when an identical set of delays was used at both tapping rates. Experiment 3 also provided further confirmation that the critical interval closely matches the tapping rate.

\section{GENERAL DISCUSSION}

The present work has established two important empirical results for DAF. First, there is clear evidence for a critical interval in tapping - that is, for a delay value that causes maximal impairment. Second, this critical in- 
terval is not fixed but depends on the tapping rate and is, in fact, approximately equal to it. These results have a number of implications for theories of DAF.

\section{Implications for Feedback Theories}

The shift in critical interval with tapping rate is incompatible with at least one feedback-based explanation for the critical interval. One interpretation for the decreasing impairment found with higher delay values is that the perceptual-motor system has a fixed time period within which feedback must arrive in order to be used as control information; information arriving after this window will tend not to be associated with the action that caused it. Under this proposal, the decreasing DAF impairment found with longer delays occurs when the returning feedback falls outside such a window. The present data demonstrate that if there is such a time window, it does not have a constant value; rather, it must be sensitive to production rate. Such a requirement will complicate any such feedback hypotheses.

Closed-loop explanations face an additional problem: Auditory feedback appears to be unnecessary for adequate performance in tapping (Chase, Harvey, et al., 1961; Finney, 1999) or music (Finney, 1997; Gates \& Bradshaw, 1974; although see Repp, 1999). Even in speech, auditory feedback deprivation resulting from acquired deafness results in much subtler impairments than have been found with DAF (Waldstein, 1990). Feedback explanations must therefore take into account the fact that feedback information is not necessary for adequate performance, although when such information is present (at least when delayed), it has an obligatory influence.

\section{Implications for Other Theories}

The NST of MacKay (1987) and the DRH of Howell et al. (1983) make contrasting predictions about rate effects on the critical interval. The NST account of DAF impairment proposes that DAF effects (in particular, repetitions) occur because the delayed feedback arrives during a "hyperactive" phase of the mental node that caused the action; this returning feedback causes reactivation of that node and a repetition of the initial act. For a given person and a given behavior, the hyperactivity phase is assumed to be at a relatively fixed time after initial node activation, and this fixed time corresponds to the critical interval. Since production rate has no effect on the timing of the hyperactive phase (at least in the theory as described in MacKay, 1987), rate should have no effect on the critical interval, consistent with the findings of MacKay (1968) for speech. Such an explanation clearly cannot apply to the tapping data, where the critical interval changes with performance rate; the present finding thus appears to be inconsistent with the current formulation of the NST. One possibility is that DAF impairment in tapping requires a fundamentally different explanation than DAF impairment in speech, although the similarities in impairment across the two domains would then be unexplained. Alternatively, the NST could be modified so that the activation cycle of a node is dependent on production rate. The decision as to which of these two approaches to take relies crucially on a better empirical understanding of whether there is a critical interval shift in speech.

The DRH of Howell et al. (1983) claims that DAF disruption occurs because of a particularly impairing rhythm set up by the overlap of the delayed feedback with some form of undelayed feedback (or with the action itself). The timing relationship that is particularly impairing is one in which the onset of delayed feedback from an action coincides with the offset of the undelayed feedback for that action. Changing the length of a produced unit (e.g., a syllable) will change the time of offset for that unit, which will lead to a change in critical interval. The data of Robinson (1972), which show an effect of speech production rate on critical interval, were cited by Howell et al. as consistent with the DRH. The dependence of critical interval on tapping rate in our experiments is consistent with this aspect of the DRH; in addition, the matching of critical interval and tapping rate suggests that the unit that is relevant to the DRH is the individual tap, rather than a higher level grouping.

Both the NST and the DRH make additional and specific timing predictions. In the NST proposal, the repetitions under DAF are directly caused by the returning feedback; therefore, the (delayed) feedback must precede the repetition. In the DRH, maximal impairment occurs when the (delayed) feedback onset for a unit coincides with the synchronous feedback offset for a previous unit. Some preliminary tapping data that are relevant to these predictions are provided in Finney (1999), although space limitations preclude their discussion here.

\section{Generality of Results}

One important question is the generalizability of the rate effect on critical interval in tapping to other forms of DAF impairment. Many researchers have assumed that DAF effects in tapping and speech reflect the same phenomenon; writings on DAF (e.g., Chase, Harvey, et al., 1961; Howell et al., 1983; Lee, 1950; MacKay, 1987) often have discussed both speech and such tasks as tapping or Morse code without making any explicit differentiation between them. The existence of a DAF critical interval in tapping is a significant analogue to the DAF effect in speech. However, the present work has also demonstrated that the critical interval in tapping depends on production rate, whereas there have been mixed results with regard to this question in the speech literature (MacKay, 1968; Robinson, 1972). Clearly, the tapping results will be of more interest if they are relevant to other domains and reflect on general theories of feedback and perception/action coupling.

There are certainly a number of differences between speech and tapping. Speech includes both air- and boneconducted sound, and the speech DAF task (in which only the air-conducted sound can be manipulated) thus necessarily involves the combination of synchronous and delayed auditory feedback. Our tapping task had delayed 
auditory feedback, but no synchronous auditory feedback (although synchronous feedback was available from other channels-e.g., tactile and proprioceptive); a conflict between synchronous and delayed feedback in the same modality may be relevant to DAF effects. A second potentially important factor is that speech is a continuous stream of constantly varying units (e.g., syllables), whereas tapping of a single finger is a sequence of discrete and similar units (finger taps) that are differentiated primarily by their placement in time. Unit identity might play an important role in the process of DAF impairment (as is suggested by feedback theories, although see Howell et al., 1983).

If future research finds a reliable rate effect on the critical interval in speech, it would speak to an additional strong similarity between speech and tapping and to the generality of the results here. If further work establishes that there are no rate effects on critical interval in speech, it would suggest that there is an important difference between the two domains and a possible need for a partially domain-specific explanation of DAF effects. Further research on DAF and music performance might also be useful: Like tapping, music tasks allow precise control of timing, but music performance also allows detailed analysis of error subtypes to a degree not possible in finger tapping (see, e.g., Palmer \& van de Sande, 1993).

\section{Complexity}

As is stated in note 1, Chase, Rapin, Gilden, Sutton, and Guilfoyle (1961) and Finney (1999) have reported that simple isochronous tapping shows little or no impairment under DAF, and Chase, Rapin, et al. have suggested that the degree of DAF impairment in tapping is modulated by the complexity of the pattern. Two results from the present experiments corroborate this viewpoint. In Experiments 1 and 3, which used a 4-2 pattern, more errors occurred with the 4-tap group than with the 2-tap group; a longer group may constitute a more complex pattern than a shorter group. In addition, a comparison of Experiments 1 and 2 or of Experiments 2 and 3 (see Table 1) shows that more errors occurred with the 4-2 pattern than with the 3-tap pattern; a pattern of two different parts is presumably more complex than a pattern consisting of a single part. In fact, complexity effects of various sorts have been repeatedly reported for DAF. In speech, MacKay (1987) and Chase (1958) reported an absence of DAF impairment when a single syllable was repeated, in contrast to the dramatic impairment in continuous speech (although see Fletcher \& Yates, 1971; Lee, 1950). For Morse code, Howell et al. (1983) noted that Morse code letters including a mixture of dots and dashes were more impaired under DAF than were patterns containing only one type of unit, and Yates (1965) reported more errors with Morse code letters involving three or four units (dots and dashes) than with letters containing only one or two. Smith (1966) reported that performance of difficult musical pieces was more impaired under DAF than were simpler musical pieces. Although such observations regard- ing complexity are descriptive rather than explanatory, they suggest a worthwhile area for further investigation of DAF and another source of possible differences between speech and tapping.

\section{Clocks, Oscillators, and Perceptual-Motor Synchronization}

Understanding the process by which DAF impairs rhythmic tapping may require a detailed theory of how such rhythmic patterns are generated. The insertion timing data of Experiments 1, 2, and 3 suggest that one aspect of the production of such patterns is a clock or oscillator that generates the base time interval underlying the pattern (e.g., at the 250-msec tapping rate, the 250-msec interval is a common denominator of both the $250-\mathrm{msec}$ within-group interval and the 500-msec between-group interval). In all three experiments, insertion errors were consistently made in time with such an underlying beat. That is, even when performance of the patterns was severely disrupted by DAF and a group was incorrectly produced with an extra tap, this extra tap maintained the underlying beat. Such consistency of timing could be interpreted in terms of the timing nodes of the NST (MacKay, 1987); alternatively, it could be viewed as a form of oscillator consistent with dynamical systems theory (e.g., Large \& Jones, 1999).

In fact, one of the most striking aspects of the present results is the close match between the critical interval and the tapping rate. The subjects made the most errors when the auditory feedback approximately coincided with the next tap. This coincidence of sound and action at the delay of maximal impairment is consistent with earlier proposals that errors under DAF are an attempt to reduce the discrepancy between feedback and action and suggests the possibility that subjects may be inserting taps to match the anticipated feedback. In the speech literature, Goldiamond, Atkinson, and Bilger (1962) suggested that behavior in DAF might result from an attempt to cause maximal overlap between feedback and action, and Fairbanks and Guttman (1958) observed that DAF repetitions may serve to restore "normal phase relationships" (p. 20). Yates (1963b) noted that abnormal feedback such as DAF leads to an attempt to "restore the usual relationship" (p. 114) of synchronized feedback and action, and Howell et al. (1983, p. 776) suggested that one strategy for overcoming DAF effects in tapping or Morse code tasks may be to synchronize the motor acts with the sound being produced. In the more specific proposal of Harrington (1988), the impaired speech behavior under DAF occurs because spoken syllable nuclei are produced to correspond with the expected (delayed) syllable nuclei.

We suggest that a dynamical systems view is a promising approach that merges these previous ideas with current theoretical concepts. For example, in the tapping DAF task, a pattern-generating oscillator could become synchronized with or entrained to the rhythm of the delayed feedback signals. Such an approach could account for the critical interval results of the present experiment: 
An alteration in the tapping pattern (such as an insertion) might occur only when the entraining feedback is closely matched in frequency and phase to the pattern generator, consistent with the matching of critical interval to tapping rate. Whether such an entrainment proposal can be further developed to fully account for the tapping data (current theories do not address the production of gapped patterns) and whether such a proposal would have relevance for DAF and speech are questions for future research. In any case, the present evidence that the critical interval in rhythmic tapping varies with the performance rate provides a challenge for theories of DAF effects.

\section{REFERENCES}

Black, J. (1951). The effect of delayed side-tone upon vocal rate and intensity. Journal of Speech \& Hearing Disorders, 16, 56-60.

Butler, R. A., \& Galloway, F. T. (1957). Factoral analysis of the delayed speech feedback phenomenon. Journal of the Acoustical Society of America, 29, 632-635.

CHASE, R. (1958). Effect of delayed auditory feedback on the repetition of speech sounds. Journal of Speech \& Hearing Disorders, 23, 583590.

CHASE, R. (1965). An information-flow model of the organization of motor activity I: Transduction, transmission, and central control of sensory information. Journal of Nervous \& Mental Disease, 140, 239-251.

Chase, R., Harvey, S., Standfast, S., Rapin, I., \& Sutton, S. (1959). Comparison of the effects of delayed auditory feedback on speech and key tapping. Science, 129, 903-904.

Chase, R., Harvey, S., Standfast, S., Rapin, I., \& Sutton, S. (1961). Studies on sensory feedback I: Effect of delayed auditory feedback on speech and keytapping. Quarterly Journal of Experimental Psychology, 13, 141-152.

Chase, R, Rapin, I., Gilden, L., Sutton, S., \& Guilfoyle, G. (1961). Studies on sensory feedback II: Sensory feedback influences on keytapping motor tasks. Quarterly Journal of Experimental Psychology, 13, 153-167.

Fabbro, F., \& Daro, V. (1995). Delayed auditory feedback in polyglot simultaneous interpreters. Brain \& Language, 45, 309-319.

FAIRBANKS, G. (1954). Systematic research in experimental phonetics 1: A theory of the speech mechanism as a servosystem. Journal of Speech \& Hearing Disorders, 19, 133-139.

FAIRBANKS, G. (1955). Selective vocal effects of delayed auditory feedback. Journal of Speech \& Hearing Disorders, 20, 333-346.

Fairbanks, G., \& GutTman, N. (1958). Effects of delayed auditory feedback upon articulation. Journal of Speech \& Hearing Research, 1, 12-22.

FinNEY, S. A. (1997). Auditory feedback and musical keyboard performance. Music Perception, 15, 153-174.

Finney, S. A. (1999). Disruptive effects of delayed auditory feedback on motor sequencing. Unpublished doctoral dissertation, Brown University.

FinNEY, S. A. (2001). FTAP: A Linux-based program for tapping and music experiments. Behavior Research Methods, Instruments, \& Computers, 33, 65-72.

Fletcher, J. F., \& Yates, A. J. (1971). The repetition of single speech sounds under delayed auditory feedback. British Journal of Psychology, 62, 73-79.

Gates, A., \& Bradshaw, J. L. (1974). Effects of auditory feedback on a musical performance task. Perception \& Psychophysics, 16, 105-109.

Gates, A., Bradshaw, J. L., \& Nettleton, N. C. (1974). Effect of different delayed auditory feedback intervals on a music performance task. Perception \& Psychophysics, 15, 21-25.

Gibson, J. J. (1966). The senses considered as perceptual systems. Boston: Houghton Mifflin.

Goldiamond, I., At kinson, C., \& Bilger, R. (1962). Stabilization of behavior and prolonged exposure to delayed auditory feedback. Science, 135, 437-438.

HARrington, J. (1988). Stuttering, delayed auditory feedback, and linguistic rhythm. Journal of Speech \& Hearing Research, 31, 36-47.

HAV LICEK, L. (1968). Effects of delayed auditory feedback on musical performance. Journal of Research in Music Education, 16, 308-318. Helson, H. (1964). Adaptation-level theory: An experimental and systematic approach to behavior. New York: Harper \& Row.

Howell, P., Powell, D., \& Khan, I. (1983). Amplitude contour of the delayed signal and interference in delayed auditory feedback tasks. Journal of Experimental Psychology: Human Perception \& Performance, 9, 772-784.

Huggins, A. (1968). Delayed auditory feedback and the temporal properties of the speech material. Zeitschrift für Phonetik, Sprachwissenschaft, und Kommunikations-Forschung, 21, 53-60.

Kalmus, H., Denes, P., \& Fry, D. (1955). Effect of delayed acoustic feedback on some non-vocal activities. Nature, 175, 1078.

Karlovich, R, \& Graham, J. (1966). Effects of pure tone synchronous and delayed auditory feedback on keytapping performance to a programmed visual stimulus. Journal of Speech \& Hearing Research, 9, 596-603.

Karlovich, R, \& Graham, J. (1967). Visually and auditorily paced keytapping performance during synchronous, decreased, and delayed auditory and visual feedback. Journal of Speech \& Hearing Research, 10, 859-864.

LARge, E. W., \& Jones, M. R. (1999). The dynamics of attending: How people track time-varying events. Psychological Review, 106, 119-159.

LEE, B. S. (1950). Effects of delayed speech feedback. Journal of the Acoustical Society of America, 22, 824-826.

LeE, B. S. (1951). Artificial stutter. Journal of Speech \& Hearing Disorders, 16, 53-55.

LENNEBERG, E. (1967). Biological foundations of language. New York: Wiley.

MacKaY, D. G. (1968). Metamorphosis of a critical interval: Agelinked changes in the delay in auditory feedback that produces maximal disruption of speech. Journal of the Acoustical Society of America, 43, 811-821.

MACKAY, D. G. (1987). The organization of perception and action. New York: Springer-Verlag.

Massey, H. (1987). The complete DX7II. New York: Amsco.

Palmer, C., \& VAN de SANDE, C. (1993). Units of knowledge in music performance. Journal of Experimental Psychology: Learning, Memory, \& Cognition, 19, 457-470.

REPP, B. H. (1999). Effects of auditory feedback deprivation on expressive piano performance. Music Perception, 16, 409-438.

Robinson, G. (1972). The delayed auditory feedback effect is a function of speech rate. Journal of Experimental Psychology, 95, 1-5.

Ruhm, H., \& CoOper, W. (1962). Low sensation level effects of puretone delayed auditory feedback. Journal of Speech \& Hearing Research, 5, 185-193.

Siegel, G. M., Fehst, C. A., Garber, S. R., \& Pick, H. L. (1980). Delayed auditory feedback with children. Journal of Speech \& Hearing Research, 23, 802-813.

Sмiтн, K. U. (1966). Cybernetic theory and analysis of learning. In E. A. Bilodeau (Ed.), Acquisition of skill (pp. 425-482). New York: Academic Press.

WALDSTEIN, R. (1990). Effects of postlingual deafness on speech production: Implications for the role of auditory feedback. Journal of the Acoustical Society of America, 88, 2099-2114.

Wing, A. M., \& Kristofferson, A. B. (1973). The timing of interresponse intervals. Perception \& Psychophysics, 13, 455-460.

Yates, A. J. (1963a). Delayed auditory feedback. Psychological Bulletin, 60, 213-232.

Yates, A. J. (1963b). Recent empirical and theoretical approaches to the experimental manipulation of speech in normal subjects and in stammerers. Behaviour Research \& Therapy, 1, 95-119.

YATES, A. J. (1965). Effects of delayed auditory feedback on Morse code transmission by skilled operators. Journal of Experimental Psychology, 69, 467-473.

Zanini, S., Clarici, A., Fabbro, F., \& Bava, A. (1999). Speaking speed 
effects on delayed auditory feedback disruption of speech fluency. Perceptual \& Motor Skills, 89, 1095-1109.

Zimmerman, G., Brown, C., Kelso, J., Hurtig, R., \& Forrest, K. (1988). The association between acoustic and articulatory events in a delayed auditory feedback paradigm. Journal of Phonetics, 16, 437 451.

\section{NOTES}

1. As was noted by Chase, Harvey, et al. (1961), the degree of DAF impairment in tapping appears to be modulated by the complexity of the pattern, with simple isochronous tapping showing little or no impairment under DAF (Chase, Harvey, et al., 1961; Finney, 1999). This complexity issue will be raised again in the General Discussion.

2 . The white noise was generated by a minor modification for the TX81Z of the white noise voice in Massey (1987). In fact, the relatively high white noise level $(79 \mathrm{~dB})$ was not enough to stop a determined subject from tapping very hard and hearing the physical tap through the headphones. Occasionally, when subjects were tapping exceedingly hard (i.e., when they were able to hear their own keystrokes), they were asked to tap more softly; this rarely occurred.

3 . Such a measure does not take correct sequencing into accountfor example, that a 2-tap group following a 2-tap group is a form of error. Counting such sequencing errors increased the combined-errors count by only a small amount (in Experiment $1,1 \%$ for the 250 -msec tapping rate and $3 \%$ for the 400 -msec tapping rate), and so only the simpler measure is reported here.

4. A group of five taps is unambiguously a single insertion. For the 4-2 pattern, a 3-tap group could potentially be either a 4-tap group with a deletion or a 2-tap group with an insertion. This ambiguity was resolved on the conservative side by classifying a 3-tap group as an insertion on a 2-tap group only if it followed a group of four or more taps; otherwise, it was counted as a miscellaneous error.

(Manuscript received December 28, 1999; revision accepted for publication November 15, 2001.) 